\title{
Hábitos alimentares do consumidor de leite cru de Campo Mourão - PR
}

\section{Alimentary habits of raw milk consumer from Campo Mourão - PR}

\author{
Luís Augusto Nero ${ }^{1 *}$; Denir Maziero²; Márcia M.S. Bezerra ${ }^{3}$
}

\begin{abstract}
Resumo
No Brasil o consumo de leite cru é bastante alto, especialmente em comunidades rurais. Grande parte da produção de leite no Brasil é comercializada sem nenhum tratamento térmico ou processamento, contradizendo a Legislação Federal, além de oferecer riscos à saúde do consumidor. Este trabalho busca traçar um perfil do consumidor de leite cru na cidade de Campo Mourão, região oeste do Paraná, Brasil, com o objetivo de fornecer informações para órgãos oficiais atuarem no controle desse mercado informal e em programas de conscientização da população. Dessa forma, foi realizado um levantamento em 423 residências da zona urbana da cidade baseado num questionário abordando o consumo de leite, consumo de leite cru e origem do leite cru recebido. Os dados coletados foram compilados e analisados (frequiências) pelo software Epi Info 2000 v 1.1 (CDC). 33,57\% da população de Campo Mourão consumia leite cru, sendo que esse hábito estava diretamente ligado a conceitos de que esse tipo de leite é mais saudável. A maioria da população consumidora de leite cru desconhecia os riscos que esse produto poderia oferecer. Na maioria dos casos, a entrega do produto era feita na residência do consumidor $(89,60 \%)$, mais freqüentemente entre 9:00 e 10:00hs (52,00\%), em garrafas plásticas tipo pet (54,00\%). A maioria da população não sabia que a venda de leite cru é proibida $(83,90 \%)$. Os resultados reforçam a necessidade de medidas efetivas de controle ao comércio de leite cru, além de evidenciar a necessidade de programas de conscientização da população em relação aos perigos que esse produto pode oferecer.
\end{abstract}

Palavras-chave: Leite cru, leite informal, hábitos alimentares, consumidor.

\begin{abstract}
In Brazil, raw milk is largely consumed, especially within rural communities. A large parcel of total Brazilian milk production is commercialized without any thermal treatment or processing, contradicting the Federal Legislation and offering risks to consumers health. This paper looks for tracing the profile of raw milk consumers from urban zone of Campo Mourão city, western Paraná State, Brazil, and objectives to give information for official organs act in control of raw milk commercialization and development of awareness programs for population. A research was effectuated in 423 houses from urban zone based in a questionnaire with questions about milk consumption, raw milk consumption and its origin. The data was compiled and analyzed by Epi Info v 1.1 software (CDC). $33.57 \%$ of population consumed raw milk, and this was linked to the idea that it is healthier. A great part of population did not know the risks of raw milk consumption. The delivery is effectuated at the consumer house (89.60\%), usually between 9:00 and 10:00 AM (52.00\%) was plastic bottles $(54.00 \%)$. The majority of population did not know that the raw
\end{abstract}

\footnotetext{
1 Doutorando do Programa de Pós-graduação em Ciência dos Alimentos - Bromatologia, Faculdade de Ciências Farmacêuticas, Universidade de São Paulo, São Paulo SP. Programa de Pós-graduação em Ciência dos Alimentos - Bromatologia (Doutorado), Faculdade de Ciências Farmacêuticas, Universidade de São Paulo. Av. Prof. Lineu Prestes, nº 580, Bloco 14 - Cidade Universitária - 05508-900 - São Paulo SP. E-mails: nero@ sercomtel.com.br e luisnero@yahoo.com.br

2 Acadêmico do curso de Medicina Veterinária, Centro Intergado de Ensino Superior, Campo Mourão PR

Prefeitura Municipal de Campo Mourão.

* Autor para correspondência.
} 
Nero, L. A.; Maziero, D.; Bezerra, M. M. S.

milk commercialization is forbidden. The results show the necessity of effective measures to control raw milk commercialization and stress the need of population awareness programs concerning the raw milk consumption risks.

Key words: Raw milk, informal milk, eating behavior, consumer.

\section{Introdução}

A população mundial tem se preocupado cada vez mais com o consumo de alimentos considerados saudáveis e seguros. Isso se deve principalmente pela ampla divulgação de surtos de Enfermidades Transmitidas por Alimentos (ETA), além de várias reportagens que constantemente aparecem na mídia sobre segurança alimentar. Apesar dessa conscientização, as ETA são ainda uma das principais causas de morbidade em diversos países (ANGELILLO et al., 2001; ANGELILLO et al., 2000; BEAN; GRIFFIN, 1990). Estima-se que os Estados Unidos gastam anualmente entre US\$ 8,4 a 10 bilhões, em cerca de 9 milhões de casos de ETA (ALTEKRUSE et al., 1996; KLONTZ et al., 1995). No Brasil existem poucas informações da real situação das ETA; os poucos dados epidemiológicos que alguns estados conseguem compilar dificilmente refletem a real situação do problema, uma vez que a maioria dos casos raramente é comunicada aos órgãos de saúde locais.

Um alimento é considerado um risco microbiológico à saúde quando está contaminado com microrganismos patogênicos. Baseado nisso, diversos países previnem as ETA procurando produzir alimentos livres de patógenos, que é conseguido através de tratamentos (pasteurização, irradiação, ozonização...), controle da contaminação na produção, processamento, transporte e estocagem (através de programas como HACCP) e conscientização da população em geral sobre práticas higiênicas de manipulação de alimentos (ALTEKRUSE et al., 1996). O consumidor final exerce papel fundamental na prevenção das ETA (JAY, COMAR, GOVENLOCK, 1999a; JAY, COMAR, GOVENLOCK, 1999b; KNABEL, 1995; SABA et al., 1998; FEIN, LIN, LEVY, 1995), uma vez que pode contaminar um alimento apenas no momento do consumo, devido à manipulação inadequada na própria residência.

Por ser um importante componente da dieta humana (SABA et al., 1998), o leite pode representar uma importante fonte de contaminação das ETA, já que sem tratamento adequado possui uma série de microrganismos patogênicos (BOOR, 1997), além de baixa qualidade microbiológica e físico-química (BELOTI et al., 1999). Apesar de proibido no Brasil desde 1952 (BRASIL, 1952), estima-se que cerca de metade da produção nacional de leite é comercializada informalmente (RIOS ESTUDOS E PROJETOS, 2002), ou seja, sem inspeção sanitária. Apesar do mercado oferecer várias opções, sabe-se que uma grande parcela da população ainda consome leite cru (RIOS ESTUDOS E PROJETOS, 2002) evidenciando a importância da opinião do consumidor nessa questão.

Portanto a adoção de medidas que evitem o consumo e a comercialização de leite cru depende do perfil do consumidor, que é quem exige esse tipo de produto, além de ser necessária a busca de opções para o destino dessa produção informal. Análises dessa natureza são mais eficientes sendo efetuadas isoladamente em pequenas regiões, já que as realidades de produção e consumo de leite cru são extremamente distintas em diferentes partes do país. Assim, o objetivo desse trabalho é fornecer informações sobre o consumo de leite cru na cidade de Campo Mourão PR, bem como as características dos consumidores desse produto, auxiliando órgãos oficiais a atuarem no controle desse comércio informal e no desenvolvimento de programas de conscientização da população. 


\section{Metodologia}

O perfil dos consumidores de leite cru da zona urbana da cidade de Campo Mourão foi obtido através de um questionário constituído basicamente de 27 questões de múltipla escolha que foi aplicado à população. Esse formulário foi formulado buscandose obter: (1) informações gerais, (2) informações sobre o consumo de leite, (3) informações sobre consumo de leite cru e (4) informações sobre a obtenção e manipulação do leite cru (Tabela 1).

Tabela 1. Perguntas/características sobre hábitos alimentares em relação ao consumo de leite e leite cru aplicadas à população da zona urbana da cidade de Campo Mourão PR, entre outubro de 200 e dezembro de 2001.

\begin{tabular}{|c|c|}
\hline \multicolumn{2}{|c|}{ 1. Informações gerais } \\
\hline & Nome e endereço \\
\hline & Escolaridade \\
\hline \multicolumn{2}{|c|}{ 2. Informações sobre o consumo de leite } \\
\hline & Consome leite? \\
\hline & Qual o destino do leite? \\
\hline & Tipo de leite que consome? \\
\hline & O consumo é diário? \\
\hline & Quantos litros consomem por dia? \\
\hline & Tem o hábito de ferver o leite antes de consumir? \\
\hline & Quanto tempo o leite permanece viável na geladeira? \\
\hline & Sabe que existem doenças transmitidas pelo leite? \\
\hline \multicolumn{2}{|c|}{ Informações sobre o consumo de leite cru } \\
\hline & Sabe o que é leite cru? \\
\hline & Consome leite cru? \\
\hline & Por que consome leite cru? \\
\hline & Qual o destino do leite cru? \\
\hline & Ferve o leite cru antes do consumo? \\
\hline & Por quanto tempo ferve o leite cru? \\
\hline & Conhece alguma doença que o leite cru pode causar? \\
\hline & Caso faça derivados, qual o destino? \\
\hline & ações sobre a origem de leite cru \\
\hline & É entregue em casa? \\
\hline & Caso não seja entregue em casa, aonde é adquirido? \\
\hline & Horário de entrega do leite cru em casa? \\
\hline & Freqüiência de entrega do leite cru? \\
\hline & Acondicionamento do leite cru? \\
\hline & O leite cru é entregue em mãos? \\
\hline & Conhece a origem do leite cru? \\
\hline & "Confia" em quem entrega/vende o leite cru? \\
\hline & Conhece alguma lei que proíba a venda de leite cru? \\
\hline
\end{tabular}

O número de entrevistas foi calculado com o auxílio do programa Epi Info 2000 v 1.1 (CENTERS FOR DISEASE CONTROL AND PREVENTION, 2000), considerando o total de 24.873 residências na zona urbana, $95 \%$ de intervalo de confiança e $10 \%$ de erro, obtendo-se o valor de 366. Entretanto, o questionário foi aplicado em 423 residências, entre outubro de 2000 e dezembro de 2001. As residências escolhidas para a aplicação do questionário foram selecionadas aleatoriamente em diferentes regiões da cidade, distribuídas de tal forma que cobrisse toda a zona urbana, incluindo bairros centrais e periféricos.

As respostas dos questionários foram compiladas e analisadas utilizando-se o programa Epi Info 2000 v 1.1 (CENTERS FOR DISEASE CONTROL AND PREVENTION, 2000), observando-se as frequiências obtidas de cada item e as relacionando com o consumo ou não de leite cru.

\section{Resultados}

Do total de 423 amostras, verificou-se que 404 $(95,51 \%)$ entrevistados possuíam o hábito de consumir leite; os principais tipos de leite consumidos nos bairros centrais e da periferia estão ilustrados na Figura 1.

A maioria dos entrevistados possuía nível de escolaridade com $1^{\circ}$ grau incompleto (230 - 54,73\%), sendo verificado a mesma distribuição em relação aos diferentes tipos de leite consumidos e aos diferentes bairros pesquisados na cidade. Dentre os consumidores de leite, $356(88,11 \%)$ o consumiam diariamente e $303(75,00 \%)$ o ferviam antes do consumo, independente do tipo. 388 (96,04\%) conservavam o leite em geladeira, que permanecia bom para o consumo, segundo os entrevistados, por até 1 dia (235 - 58,17\%), 3 dias (139-34,41\%), 5 dias $(9-2,23 \%)$ e até mesmo mais de 5 dias $(5-1,24 \%)$. Considerando todos os entrevistados, 334 (78,96\%) eram capazes de definir o que é o leite cru e $288(68,09 \%)$ sabiam que o leite podia transmitir doenças.

O consumo de leite cru foi verificado em 142 $(33,57 \%)$ residências. Dentre as razões descritas para o consumo, as principais respostas foram por considerálo "mais forte" e de menor custo (Tabela 2). 

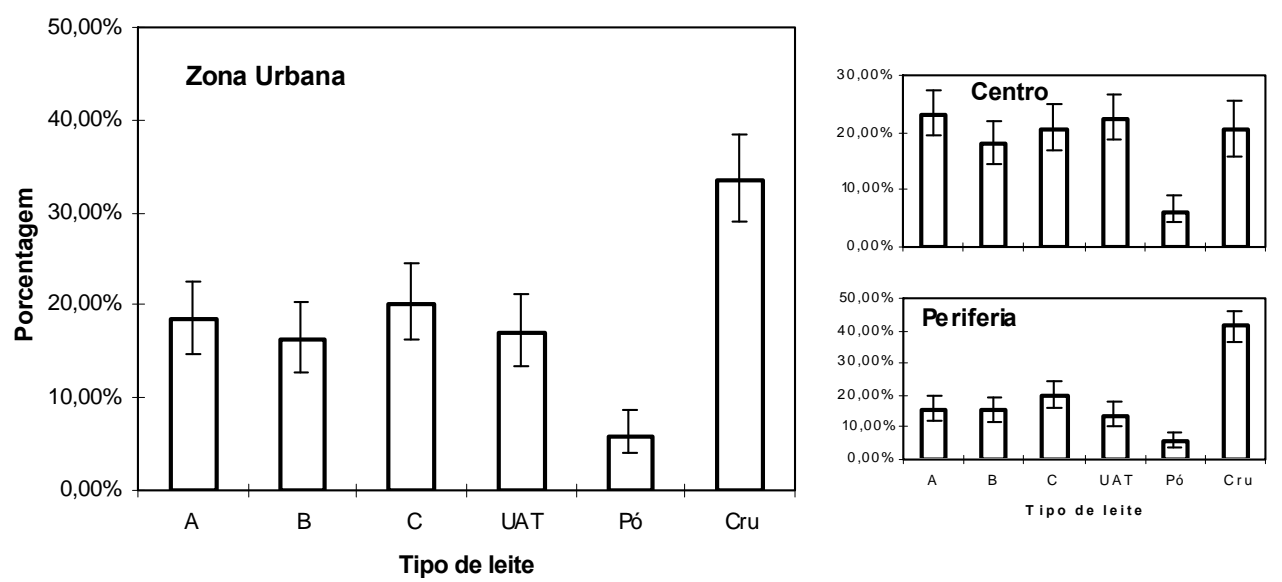

Figura 1. Tipos de leite consumidos na zona urbana (subdividida em centro e periferia) da cidade de Campo Mourão PR.

Tabela 2. Razões relatadas pelos consumidores de leite cru (142) para a escolha do produto, em Campo Mourão PR, entre setembro de 2000 e novembro de 2001.

\begin{tabular}{lcc}
\hline Razões & Freqüência (n) & Porcentagem (\%) \\
\hline Menor custo & 58 & 40,85 \\
Considerado "mais forte" & 57 & 40,14 \\
Maior praticidade & 53 & 37,32 \\
Considerado "mais puro" & 51 & 35,92 \\
Ausência de fraude (adição de água) & 12 & 08,45 \\
Única opção & 01 & 00,70 \\
\hline
\end{tabular}

A maioria dos consumidores de leite cru o utilizava como bebida (135 - 95,07\%) e $139(97,89 \%)$ entrevistados possuíam o hábito de fervê-lo antes da utilização; destes, 94 (67,63\%) desligavam a fonte de calor assim que o produto entrava em ebulição. Grande parte dos consumidores de leite cru (92 $65,20 \%$ ) desconhecia os possíveis riscos, ou não os considerava como risco, que o produto podia oferecer; os problemas ligados à saúde ou doenças mais relacionados com o consumo de leite cru foram diarréia (55 - 38,73\%), vômito (41 - 28,87\%), tuberculose (35 - 24,65\%), brucelose (30 - 21,13\%), toxoplasmose $(13-9,15 \%)$ e listeriose $(12-8,45 \%)$. 57 consumidores de leite cru $(40,14 \%)$ produziam derivados (como queijo, manteiga, doce de leite...), porém para consumo próprio.

Considerando ainda os consumidores de leite cru, $127(89,44 \%)$ entrevistados relataram que recebiam o produto na própria residência, sendo também verificado o comércio em feiras livres, na rua e em mercados. O horário de maior entrega ocorria entre 9:00 e 10:00hs (74 - 52,11\%) (Figura 2).

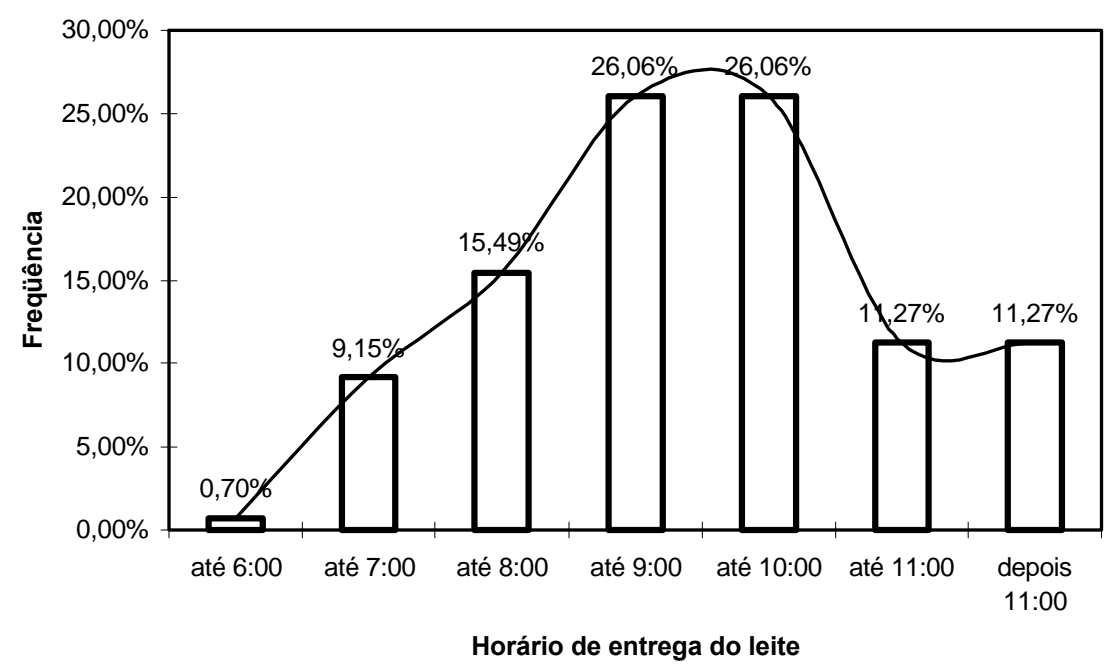

Figura 2. Horários de entrega de leite cru nas residências da zona urbana da cidade de Campo Mourão PR. 
A maioria dos consumidores recebiam o leite cru até 3 vezes por semana (56-39,44\%) que era acondicionado em garrafas plásticas tipo pet (77 $54,22 \%)$, leiteiras de metal (30 - 21,13\%), garrafas de vidro (2-1,41\%) além de outros recipientes (33 23,24\%). $136(95,78 \%)$ pessoas entrevistadas relataram que o leite cru é entregue diretamente em mãos, $82(57,70 \%)$ desconheciam o local de produção, $102(71,83 \%)$ consideravam o entregador confiável e $119(83,80 \%)$ desconheciam qualquer lei de proibição da venda de leite cru.

\section{Discussão}

Os dados dessa pesquisa revelaram que ainda existe consumo de leite cru, também denominado informal, por uma parcela considerável da população de Campo Mourão (33,57\%). Outros estudos revelaram realidades similares em diferentes regiões do país; em Pirassununga SP (OLIVAL et al., 2002) observou-se que cerca de $14 \%$ da população consumia leite fluido informal e $25 \%$ consumia queijo produzido com esse leite e em 32 cidades de São Paulo e Minas Gerais (RIOS ESTUDOS E PROJETOS, 2002) foi verificado consumo de leite cru por $65,3 \%$ da população entrevistada.

Não foi verificada nenhuma relação entre o nível de escolaridade do entrevistado e o tipo de leite consumido, o que pode descartar a relação entre grau de instrução e o consumo de leite cru. Em todos os bairros da cidade ocorria consumo de leite cru, embora fosse menor nas áreas centrais. Nos bairros da periferia da cidade foi observado maior consumo de leite cru, chegando a atingir níveis de 53,80\%, provavelmente devido ao acesso mais facilitado e pouco controlado dos distribuidores.

Considerando as razões da compra de leite cru (Tabela 2), observou-se que o consumo desse produto está ainda relacionado à crença de que é mais forte, além de ser considerado de baixo custo. Outra razão observada que estimula o consumo do leite cru é a crença de que é mais puro, além da facilidade de pagamento, uma vez que os consumidores pagavam ao produtor apenas uma vez ao mês e recebiam o produto regularmente na residência. $\mathrm{O}$ motivo do consumo de leite cru também foi verificado em estudos similares, revelando que o consumidor considera o leite cru "mais forte", "mais puro", "mais nutritivo"e com melhor sabor (OLIVAL et al., 2002; RIOS ESTUDOS E PROJETOS, 2002). Apenas 5\% dos consumidores de Pirassununga SP (OLIVAL et al., 2002) relataram que a escolha é pelo relativo baixo custo.

Apesar da maioria dos consumidores aplicarem um "tratamento térmico" ao leite (ou seja, aquecimento até o ponto de ebulição), o tempo de aplicação do calor pode ser insuficiente para garantir um produto livre de riscos. Além de não garantir a destruição de todos os microrganismos, deve-se considerar a possibilidade da produção prévia de toxinas bacterianas termoestáveis, como as enterotoxinas produzidas por Staphylococcus aureus, microrganismo presente em leite cru (BOOR, 1997). Ainda relacionado a isso, outro fator preocupante foi que a maioria dos consumidores de leite cru não considerava sua ingestão um risco à saúde.

A venda de leite cru em feiras livres e em mercados alerta para a dificuldade que órgãos oficiais de inspeção e saúde têm para fiscalizar a venda desse produto, como também foi verificado em outras localidades (BELOTI et al., 1999; OLIVAL et al., 2002; RIOS ESTUDOS E PROJETOS, 2002). Em relação ao horário de recebimento, observou-se que o leite cru ficava exposto às condições ambientais por um grande período de tempo até ser entregue no destino, uma vez que a maioria das entregas é feita em recipientes "reciclados" (garrafas plásticas tipo pet), impróprios para o armazenamento, e os veículos utilizados para entrega não possuíam recipientes refrigerados. Sabe-se que durante pequenos intervalos de tempo ocorre multiplicação bastante elevada de microrganismos, o que não garante uma boa qualidade do produto no momento da entrega.

Os resultados obtidos nessa pesquisa permitem concluir que o hábito de consumir leite cru, ou informal, por uma parcela considerável da população 
está diretamente relacionado com conceitos previamente formados de que este produto possui boa qualidade, além de desconhecimento dos riscos que esse produto pode oferecer. Portanto, punição e combate à comercialização de leite cru como medidas isoladas de controle não são suficientes para impedir o consumo, sendo fundamental o desenvolvimento de programas de conscientização da população em relação aos riscos que o leite cru pode oferecer.

\section{Referências}

ALTEKRUSE, S. F.; STREET, D. A.; FEIN, S. B.; LEVY, A. S. Consumer knowledge of foodborne microbial hazards and food-handling practices. Journal of Food Protection, Des Moines, v.59, p.287-294, 1996.

ANGELILLO, I. F.; VIGGIANI, N. M. A.; RIZZO, L.; BIANCO A. Food handlers and foodborne diseases: knowledge, attitudes and reported behavior in Italy. Journal of Food Protection, Des Moines, v.63, p.381$385,2000$.

ANGELILLO, I. F.; FORESTA, M. R.; SCOZZAFAVA, C.; PAVIA, M. Consumers and foodborne diseases: knowledge, attitudes and reported behavior in one region of Italy. International Journal of Food Microbiology, Amsterdam, v.64, p.161-166, 2001.

BEAN, N. H.; GRIFFIN, P. M. Foodborne disease outbreaks in the United States, 1973-1987: pathogens, vehicles, and trends. Journal of Food Protection, Des Moines, v.53, p.804-817, 1990.

BELOTI, V.; BARROS, M. A. F.; SOUZA, J. A. S.; NERO, L. A.; SANTANA, E. H. W.; BALARIN, O.; CURIAK, Y. Avaliação da qualidade do leite cru comercializado em Cornélio Procópio, Paraná: Controle do consumo e da pasteurização. Semina: Ciências Agrárias, Londrina, v.20, p.12-15, 1999.

BOOR, K. J. Pathogenic microorganisms of concern to the dairy industry. Dairy, Food and Environmental Sanitation, Ames, v.17, p.714-717, 1997.
BRASIL. Ministério da Agricultura, do Abastecimento e da Reforma Agrária. Departamento Nacional de Inspeção de Produtos de Origem Animal. Regulamento de Inspeção Industria e Sanitária de Produtos de Origem Animal. Aprovado pelo Decreto 30.691 de 29 de março de 1952 e alterado pelo Decreto 1.255 de 25 de junho de 1962. Rio de Janeiro. 1952.

CENTERS FOR DISEASECONTROL ANDPREVENTION. EpiInfo 2000. Disponível em: <http://www.cdc.gov/ epiinfo>. Acesso em: 6 nov. 2002.

FEIN, S. B.; LIN, C. T. J.; LEVY, A. S. Foodborne illness: perceptions, experience, and preventive behaviors in the United States. Journal of Food Protection, Des Moines, v.58, p.1405-1411, 1995.

JAY, L. S.; COMAR, D.; GOVENLOCK, L. D. A national Australian safety telephone survey. Journal of Food Protection, Des Moines, v.62, p.921-928, 1999a.

A video study of Australian domestic foodhandling practices. Journal of Food Protection, Des Moines, v.62, p.1285-1296, 1999b.

KLONTZ, K. C.; TIMBO, B.; FEIN, S.; LEVY, A. Prevalence of selected food consumption and preparation behaviors associated with increased risks of food-borne disease. Journal of Food Protection, Des Moines, v.58, p.927930, 1995.

KNABEL, S. J. Foodborne illness: role of home food handling practices. Food Technology, Chicago, v.4, p.119$131,1995$.

OLIVAL, A. A.; SPEXOTO, A. A.; CAMPOS, D. F. S.; FERREIRA, F.; FONSECA, L. F. L.; SANTOS, M. V.; DIAS, R. A. Hábitos de consumo do leite informal, associados ao risco de transmissão de doenças, no município de Pirassununga, SP. Higiene Alimentar, São Paulo, v.16, n.102/103, p. 35-40, 2002.

RIOS ESTUDOS EPROJETOS. O consumo do leite informal no Brasil. Tetrapak. Disponível em <http:// www.tetrapak.com.br>. Acesso em: 6 nov. 2002.

SABA, A.; MONETA, E.; NARDO, N.; SINESIO, F. Attitudes, habit, sensory and liking expectation as determinants of the consumption of milk. Food Quality and Preference, Barking, v.9, p.31-41, 1998. 